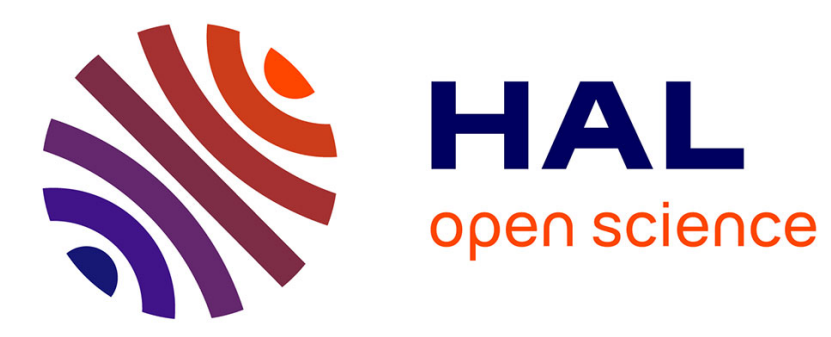

\title{
Interplanetary dust detection by radio antennas: Mass calibration and fluxes measured by STEREO/WAVES
}

\author{
A. Zaslavsky, N. Meyer-Vernet, I. Mann, A. Czechowski, K. Issautier, G. Le \\ Chat, F. Pantellini, K. Goetz, M. Maksimovic, S. Bale, et al.
}

\section{- To cite this version:}

A. Zaslavsky, N. Meyer-Vernet, I. Mann, A. Czechowski, K. Issautier, et al.. Interplanetary dust detection by radio antennas: Mass calibration and fluxes measured by STEREO/WAVES. Journal of Geophysical Research Space Physics, 2012, 117 (A5), pp.n/a-n/a. 10.1029/2011JA017480 . hal02883409

\section{HAL Id: hal-02883409 \\ https://hal.science/hal-02883409}

Submitted on 4 Nov 2021

HAL is a multi-disciplinary open access archive for the deposit and dissemination of scientific research documents, whether they are published or not. The documents may come from teaching and research institutions in France or abroad, or from public or private research centers.
L'archive ouverte pluridisciplinaire HAL, est destinée au dépôt et à la diffusion de documents scientifiques de niveau recherche, publiés ou non, émanant des établissements d'enseignement et de recherche français ou étrangers, des laboratoires publics ou privés. 


\title{
Interplanetary dust detection by radio antennas: Mass calibration and fluxes measured by STEREO/WAVES
}

\author{
A. Zaslavsky, ${ }^{1,2,3}$ N. Meyer-Vernet, ${ }^{2}$ I. Mann, ${ }^{4}$ A. Czechowski, ${ }^{5}$ K. Issautier, ${ }^{2}$ G. Le Chat, ${ }^{2}$ \\ F. Pantellini, ${ }^{2}$ K. Goetz, ${ }^{6}$ M. Maksimovic, ${ }^{2}$ S. D. Bale, ${ }^{7}$ and J. C. Kasper ${ }^{1,3}$ \\ Received 21 December 2011; revised 3 March 2012; accepted 5 April 2012; published 23 May 2012.
}

[1] We analyze dust impacts recorded by the S/WAVES radio instrument onboard the two STEREO spacecraft near 1 A.U. during the period 2007-2010. The impact of a dust particle on a spacecraft produces a plasma cloud whose associated electric field can be detected by on-board electric antennas. For this study we use the electric potential time series recorded by the waveform sampler of the instrument. The high time resolution and long sampling times of this measurement enable us to deduce considerably more information than in previous studies based on the dynamic power spectra provided by the same instrument or by radio instruments onboard other spacecraft. The large detection area compared to conventional dust detectors provides flux data with a better statistics. We show that the dust-generated signals are of two kinds, corresponding to impacts of dust from distinctly different mass ranges. We propose calibration formulas for these signals and show that we are able to use S/WAVES as a dust detector with convincing results both in the nanometer and micrometer size ranges. In the latter, the orbital motion of the spacecraft enables us to distinguish between interstellar and interplanetary dust components. Our measurements cover the mass intervals $\sim 10^{-22}-10^{-20} \mathrm{~kg}$ and $\sim 10^{-17}-5 \times 10^{-16} \mathrm{~kg}$. The flux of the larger dust agrees with measurements of other instruments on different spacecraft.

Citation: Zaslavsky, A., et al. (2012), Interplanetary dust detection by radio antennas: Mass calibration and fluxes measured by STEREO/WAVES, J. Geophys. Res., 117, A05102, doi:10.1029/2011JA017480.

\section{Introduction}

[2] Since the first space exploration missions, in-situ interplanetary dust detection has been an important issue. Most of these measurements have been performed with instruments specifically designed to characterize dust particles [Grün et al., 1992; Srama et al., 2004], but relevant studies have also been produced with the help of radio receivers that were revealed to be able to (although not designed to) measure electric signals associated with individual dust grains impacting the spacecraft body at high speed [see, e.g., Meyer-Vernet, 2001] and references

\footnotetext{
${ }^{1}$ Harvard-Smithsonian Center for Astrophysics, Cambridge, Massachusetts, USA.

${ }^{2}$ LESIA, Observatoire de Paris-CNRS-Université Pierre et Marie CurieUniversité Denis Diderot, Meudon, France.

${ }^{3}$ NASA Lunar Science Institute, Moffett Field, California, USA.

${ }_{5}^{4}$ Belgian Institute for Space Aeronomy, Brussels, Belgium.

${ }^{5}$ Space Research Center, Polish Academy of Sciences, Warsaw, Poland.

${ }^{6}$ School of Physics and Astronomy, University of Minnesota, Minneapolis, Minnesota, USA.

${ }^{7}$ Space Science Laboratory, University of California, Berkeley, California, USA.

Corresponding author: A. Zaslavsky, LESIA, Observatoire de ParisCNRS-Universit Pierre et Marie Curie-Universit Denis Diderot, 5 Place Jules Janssen, F-92195 Meudon, France. (arnaud.zaslavsky@obspm.fr)

Copyright 2012 by the American Geophysical Union. 0148-0227/12/2011JA017480
}

therein). In this article, we present the results of an analysis belonging to this latter category.

[3] STEREO consists of two spacecraft at around 1 A.U. from the Sun, in orbits that lead (STEREO A) and trail (STEREO B) the Earth. The primary objective of the STEREO mission, launched in 2006 , is to make stereoscopic observations of the solar corona and solar eruptions across a broad range of wavelength [Kaiser et al., 2008]. The S/WAVES instrument, composed of three orthogonally mounted electric antennas connected to an electronic analyzer, is designed to provide observations in the radio frequency range $(2.5 \mathrm{kHz}-16 \mathrm{MHz})$. Typical impulsive signals recorded by the instrument, sometimes with an extremely high rate, have been interpreted as being produced by nanodust impacts in a recent study based on the S/WAVES Low Frequency Receiver(LFR) data [Meyer-Vernet et al., 2009a]. The detection of larger (10 microns) dust grains impacting the STEREO spacecraft has also been reported by St.Cyr et al. [2009], using the data from the white-light instruments of the SECCHI suite. They showed that $94 \%$ of the events detected by SECCHI were associated with strong S/WAVES impulsive signals.

[4] In this article, we go further in the analysis of the STEREO dust impacts, using the S/WAVES waveform data produced by the TDS (Time Domain Sampler) subsystem of the instrument. TDS produces a time series of the voltage at the antennas terminals instead of a time integrated power 


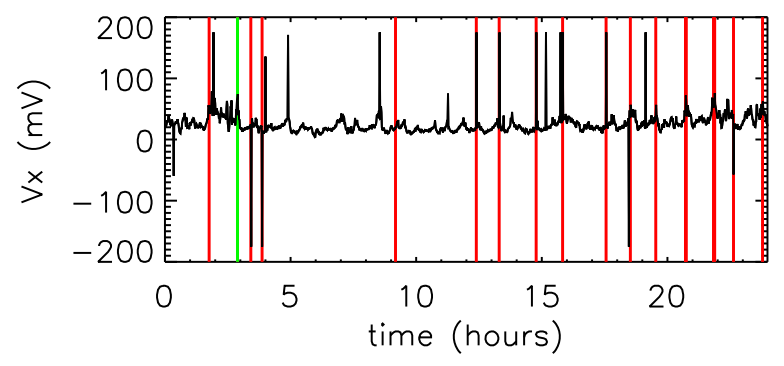

Figure 1. TDSmax signal detected on the $\mathrm{X}$ monopole of STEREO A during a period rich in dust impacts (1 January, 2009). The red lines show the times during which events were sampled. The green line shows the time where the event presented in Figure 2 was sampled.

spectrum. In section 2, after a brief presentation of the instrument, we show that two populations of dust impacts can be identified within the TDS data, and provide an interpretation and a calibration technique linking the signals amplitude to the mass of the impinging dust grains. In section 3 , we use this calibration to perform interplanetary dust flux measurements in the nanometer and micrometer ranges. Section 4 summarizes and discusses the obtained results.

\section{Dust Impact Detection by S/WAVES TDS}

[5] In this section, we present typical impulsive voltage signals detected by the TDS waveform sampler. As similar voltage pulses have been detected (directly or through their spectral signature) on a number of space missions with various radio and plasma wave instruments in several dusty environments, and were interpreted as dust impacts [Aubier et al., 1983; Gurnett et al., 1983; Meyer-Vernet et al., 1986; Oberc, 1990; Tsintikidis et al., 1995; Gurnett et al., 1997; Tsurutani et al., 2003; Kurth et al., 2006], we assume that these pulses are generated by impacts of fast dust particles on the spacecraft. The high sensitivity, time resolution and sampling time of the S/WAVES TDS, as compared to the instruments used in these previous studies, enables us to perform a very careful analysis of these signals at 1 A.U. Section 2.4 provides a physical explanation for the generation of these signals by dust impacts, according to which a link between the amplitude of the recorded signals and the mass of the impinging grains is derived.

\subsection{Presentation of the Instrument}

[6] STEREO/WAVES or S/WAVES is the radio and plasma waves experiment aboard the twin STEREO spacecraft. It is designed to perform electric field measurements in the interplanetary medium, and is presented in detail by Bougeret et al. [2008]. The S/WAVES electric field sensors are formed by three orthogonal, 6 meter, monopole antennas $\mathrm{X}, \mathrm{Y}$ and $\mathrm{Z}$ (described in detail in Bale et al. [2008]). The sensors are connected to an analyzer, that produces power spectra of the electric field (LFR and HFR modes) as well as broadband electric field waveforms (Time Domain Sampler, or TDS). In this article we make use of the data provided by the TDS to study the voltage variations measured by the instrument when dust particles are impacting the spacecraft.
[7] To this purpose, we use two TDS data sets: (1) the TDSmax data gives the maximum amplitude or peak signal detected on the antennas each minute. (2) The TDS Events data set provides full voltage time series captured by the instrument with a sampling rate of a few $\mu$ s. The time duration of a complete event is generally $\sim 65 \mathrm{~ms}$ or $\sim 130 \mathrm{~ms}$. Figure 1 shows an example of one day of TDSmax, and an event sampled during this day is presented in Figure 2. It can be seen that TDS events are mostly recorded during the periods of highest values of the TDSmax. This is due to the fact that the instrument assigns a quality factor (essentially increasing with the amplitude of the waveform) to each event, and telemeters back to Earth those events with the highest quality factor. It must thus be noted that the analysis of the data provided by the Event mode induces a bias in the statistics, that will be discussed in the following.

\subsection{Characterization of Dust Impact Signals}

[8] The systematic observation of the TDS events electric waveforms shows a broad variety of signals, with very distinctive shapes. These signals include electric potential fluctuations induced by density inhomogeneities in the local plasma, different kind of plasma waves, oscillating at the local plasma frequency (Langmuir waves), or at smaller frequencies (e.g. whistler waves), as well as interplanetary shock associated electric fields. Electric signals can also be generated by the impact of energetic particles (protons and electrons) from solar or galactic origin. All these signals are well known, identified and broadly studied by the radio and plasma waves community [see, e.g., Kellogg et al., 1996; Bale et al., 1998; Henri et al., 2011; Malaspina et al., 2011].

[9] The TDS events also include a much less studied, although very frequently observed, type of signals. Two typical examples of such signals are provided in Figure 3. These signals are characterized by a steep rise (tens of $\mu \mathrm{s}$ ) followed by a quick ( $50 \mu \mathrm{s}-1 \mathrm{~ms})$ relaxation of the electric potential to zero (for a discussion of these signals and the associated timescales, [see, e.g., Meyer-Vernet et al., 2009b]. Another characteristic of these signals is the generation of nearly simultaneous (with an accuracy of $\sim 10 \mu \mathrm{s}$ ) pulses on the three monopoles.

[10] In this article, we assume that these signals are generated by dust impacts on the spacecraft body. As will be seen in the following, this assumption is strongly supported by the obtained results, and by the physical modeling of the signal generation. Therefore, we will through the article refer to these voltage pulses as dust impact signals. Only these

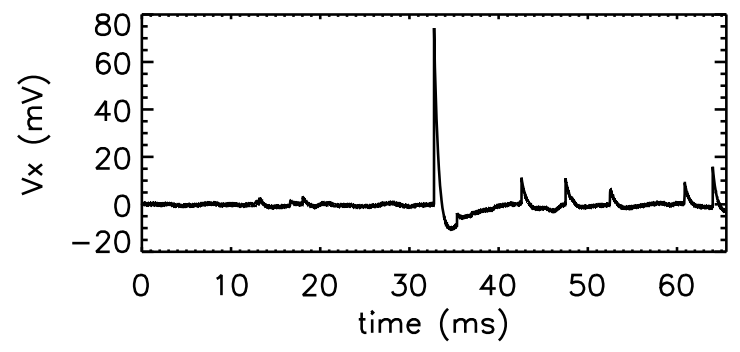

Figure 2. Sampled electric signal on the $X$ monopole of STEREO A during one of the TDS events collected on 1 January, 2009 (green line on Figure 1). 

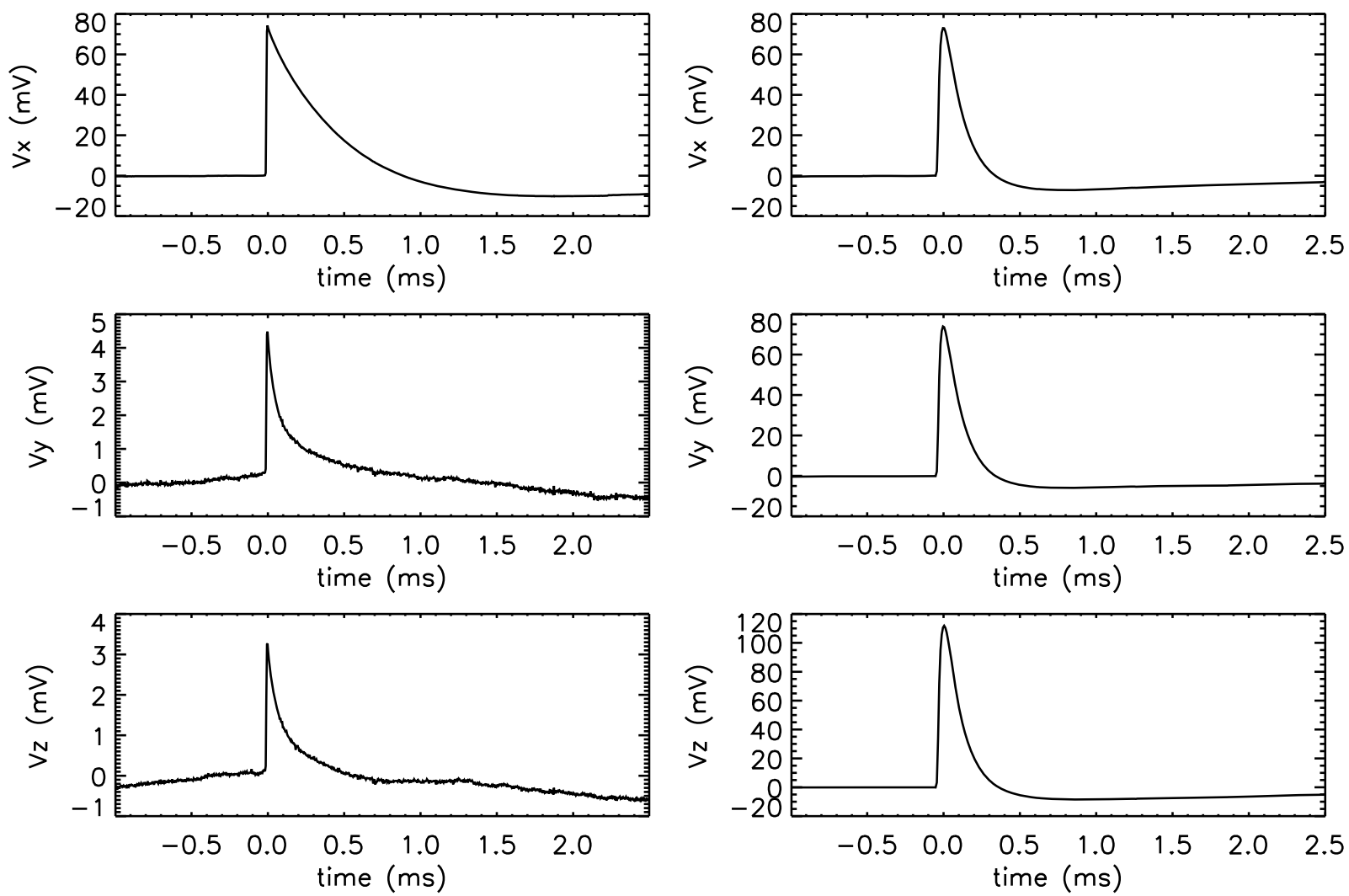

Figure 3. Typical examples of voltage impulses observed by the TDS onboard STEREO A. (top to bottom) The voltage observed on the $\mathrm{X}, \mathrm{Y}$ and $\mathrm{Z}$ monopoles. The timescale is shifted so that the time is 0 when the signal reaches its maximum on the $X$ monopole. (left) An example of a single hit (1 January, 2009, 2:54 UT). (right) An example of a triple hit (31 March, 2007, 14:48 UT) (see section 2.3 for the definition of the single and triple hits, and sections 2.4.2 and 2.4.3 for their physical interpretation).

signals have been considered in the data analysis presented hereafter.

[11] To conclude this discussion about impact signal characterization, let us note that the possibility of such signals to be generated by internal noise (jitter, power switches, noise from moving parts) as well as electromagnetic interferences from other instruments, has been extensively investigated through ground and in-flight tests performed by the S/WAVES engineering and scientific team. No evidence that these signals could have been generated by any internal noise has ever been found.

\subsection{Statistical Study and Identification of Two Populations of Impacts}

[12] Figure 1 shows the TDSmax voltage as a function of time during a day rich in dust impacts. One can see that the maximum amplitude of the electric potential rarely takes values below around $30 \mathrm{mV}$. A comparison with a quiet day would show the TDSmax much closer to zero. This means that at least a $30 \mathrm{mV}$ dust impact signal happens every minute. We can see the occurrence of negative peaks, but due to their small proportion (6 points on 1440 in the present example) we will neglect them in the present study.

[13] Figure 2 shows the typical voltage sampled on the $\mathrm{X}$ monopole of STEREO A by the TDS during a period with high dust flux. The high number of impacts detected within
$60 \mathrm{~ms}$ indicates how high the dust fluxes detected by STEREO in the interplanetary medium can be.

[14] We analyzed the TDS events recorded during the period 2007-2010, and gathered in a database all the dust impact signals observed. We limited ourselves to signals that are positive on the three monopoles, and not "complex," in the sense that the signal consists of one single isolated peak. We also limited our study to the events having an amplitude smaller than $150 \mathrm{mV}$, in order to avoid considering signals exceeding the saturation level of the instrument. The ratio of analyzed and rejected signals are summarized in Table 1.

[15] Figures 4 and 5 show the result of this study, in the form of correlations between the signal detected on each antenna. Two distinct categories of impacts are visible. The first one (that we shall refer to as "single hits") consists of a large impulse on the $\mathrm{X}$ (or Z) monopole on STEREO A (or B, respectively), and a much smaller and well correlated signal on the two other monopoles. The second category

Table 1. Summary of the Number of Dust Impacts Detected During the Period 2007-2010 in the TDS Event Data Set

\begin{tabular}{ccccc}
\hline Spacecraft & Total & $|\delta V| \leq 150(\mathrm{mV})$ & Negative & Analyzed \\
\hline STEREO A & 227263 & $203188(89 \%)$ & $19119(8 \%)$ & $163949(72 \%)$ \\
STEREO B & 103790 & $80867(78 \%)$ & $4411(4 \%)$ & $54605(53 \%)$ \\
\hline
\end{tabular}




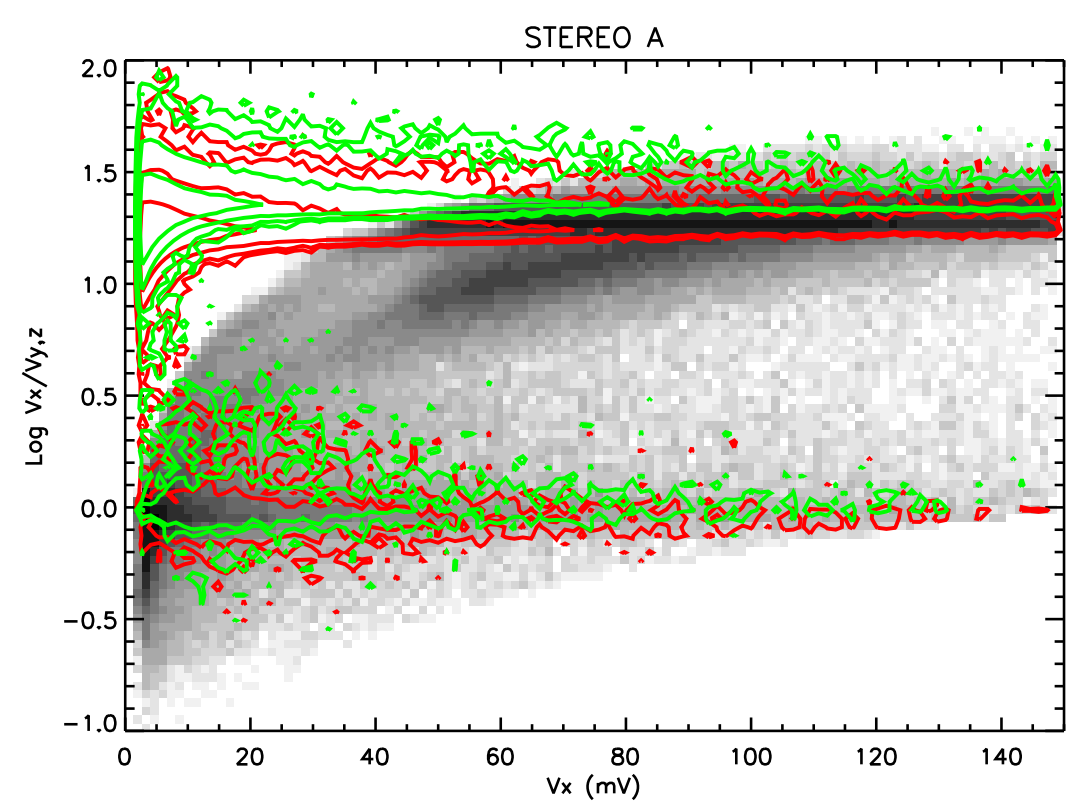

Figure 4. Ratios of the dust impact signal amplitude on the monopole $\mathrm{X}$ to that on $\mathrm{Y}$ and $\mathrm{Z}$, as a function of the amplitude on the monopole X on STEREO A. The logarithm of the TDSmax data points distribution function is represented as grey levels (arbitrary units, the darker the higher). Contour levels of the logarithm of the TDS events data points distribution function are plotted in red $\left(V_{x} / V_{y}\right)$ and green $\left(V_{x} / V_{z}\right)$. One can clearly see that two categories of dust signals are coexisting: one for which $V_{x} \sim V_{y} \sim V_{z}$ and another for which $V_{x} \sim 20 V_{y, z}$.

("triple hits") consists of signals having the same order of magnitude on the three monopoles. Figure3 shows the typical shape of voltage pulses for single and triple hits.

[16] One can see that small differences in the ratios $V_{x} / V_{y}$ and $V_{x} / V_{z}$ of the order of $10-20 \%$ exist. They are of the order of the expected gain difference between the monopoles [Zaslavsky et al., 2011]. A part of these differences can also be due to the impact occurring in average closer to a specific monopole, as well as to electronic effects such as cross-talk between the channels of the receiver. These figures also show that, in particular for the high values of the amplitude (which are less likely generated by other phenomena, such as plasma waves, density fluctuation and thermal noise) the

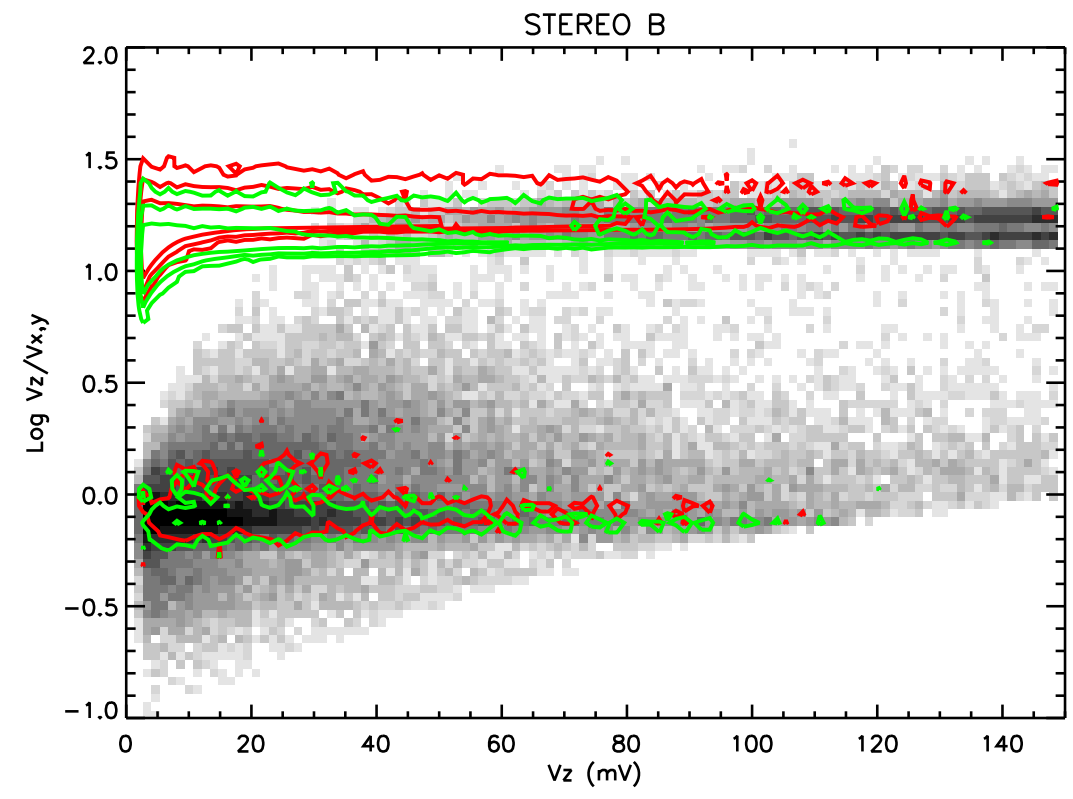

Figure 5. Same plot as Figure 4 but for STEREO B. Here are shown the ratios $V_{z} / V_{x}$ and $V_{z} / V_{y}$ (red and green respectively), as a function of the amplitude $V_{z}$. The picture is similar to the one obtained for STEREO A, with the $\mathrm{Z}$ monopole playing the role of the $\mathrm{X}$ monopole. 
Table 2. Number of Dust Impacts Detected by the S/WAVES TDS in Event Mode

\begin{tabular}{lccc}
\hline Spacecraft & Number of Events & Single Hits & Triple Hits \\
\hline STEREO A & 163949 & 157826 & 6123 \\
STEREO B & 54605 & 50088 & 4517 \\
\hline
\end{tabular}

TDSmax and TDS events data points distributions correspond closely.

[17] Table 2 presents the number of impacts, and the ratio of single and triple hits, detected in the events recorded by TDS on STEREO A and B. The number of single hits is much smaller on STEREO B than on STEREO A, whereas the number of triple hits is of the same order on the two spacecraft (it is actually somewhat larger on STEREO A, for reasons related to the difference of trajectories between the spacecraft as seen in section 3.1 and Figure 7). The second fact is that triple hits are much less frequent than single hits.

[18] In section 3, we will use the TDSmax data set to measure the flux of triple hits. This data has the advantage of presenting no gap, since TDSmax collects a voltage point per minute continuously in time. The use of a data set providing a measurement per minute only is justified by the very low probability of two triple hit impacts occurring during the same minute (as will be checked in section 3). The TDSmax does not enable us to check the peak shape of the voltage pulse, and we will have to assume that when a large voltage of the same order of magnitude is detected within the same minute on the three monopoles, it is due to a triple hit impact. This is likely, as no other frequent phenomenon is expected to produce such correlations at large amplitudes (above $\sim 15 \mathrm{mV}$ ) in the free solar wind.

[19] In contrast, as illustrated by Figure 2, the very high rate of single hit impacts makes the use of the TDS Events data set necessary for measuring their flux. Due to the previously mentioned selection criteria, the TDS events which are telemetered necessarily include at least a large amplitude peak. The flux derived from the counting of the spikes in the events will thus be biased, and constitute a higher bound on the actual flux (see the discussion of the error bars in section 3.3).

[20] To summarize, in the following all the results presented on the single-hits are obtained through the analysis of the TDS-event data, in the voltage range 3-150 $\mathrm{mV}$ (the $3 \mathrm{mV}$ bottom threshold is only here to avoid the selection of random background fluctuations). And all the results presented on the triple hits are obtained through the analysis of the TDSmax data, in the voltage range $15-150 \mathrm{mV}$.

\subsection{Physical Interpretation of the Voltage Signals}

[21] In this section, we present the physical mechanisms at the origin of the observed voltage peaks. The basis is the generation of electric charges (free electrons and ions) by a fast dust grain impacting the spacecraft body (section 2.4.1). This charge can produce a voltage pulse by the well known process of electron recollection by the spacecraft body (section 2.4.2). If a grain hits the spacecraft close enough to an antenna, the charge induced by the impact can generate a local and transient perturbation of the photoelectron equilibrium current on this antenna. This results in a strong and fast variation of this antenna's potential, even though the charge is too small to generate an important signal through electron recollection (section 2.4.3).

2.4.1. Electric Charge Generated by Impact Ionization

[22] When a dust particle hits a spacecraft with a high enough relative velocity, the grain as well as part of the target material are partially vaporized and ionized. The expansion of the resulting plasma cloud can be detected by the antennas of the spacecraft.

[23] The charge available in the cloud (i.e. the number of free electrons multiplied by the electron charge) is strongly dependent on the mass and relative velocity of the impacting dust grain. Laboratory experiments on micron-sized particles accelerated to tens of $\mathrm{km} / \mathrm{s}$ provide an empirical expression for the charge [McBride and McDonnell, 1999]

$$
Q \simeq 0.7 m^{1.02} v^{3.48}
$$

where $Q$ is expressed in Coulombs, $m$ in kilograms and $v$ in $\mathrm{km} / \mathrm{s}$. We shall use this expression despite the known uncertainties $(Q$ varies with the angle of incidence, the composition of the impinging particle and the target material). In the absence of studies dealing with fast and small grains, we shall make the strong hypothesis that this relationship is valid from nanometer to micrometer sized grains, and for velocities up to $350 \mathrm{~km} / \mathrm{s}$. Note that no instrument has ever been calibrated for such small masses

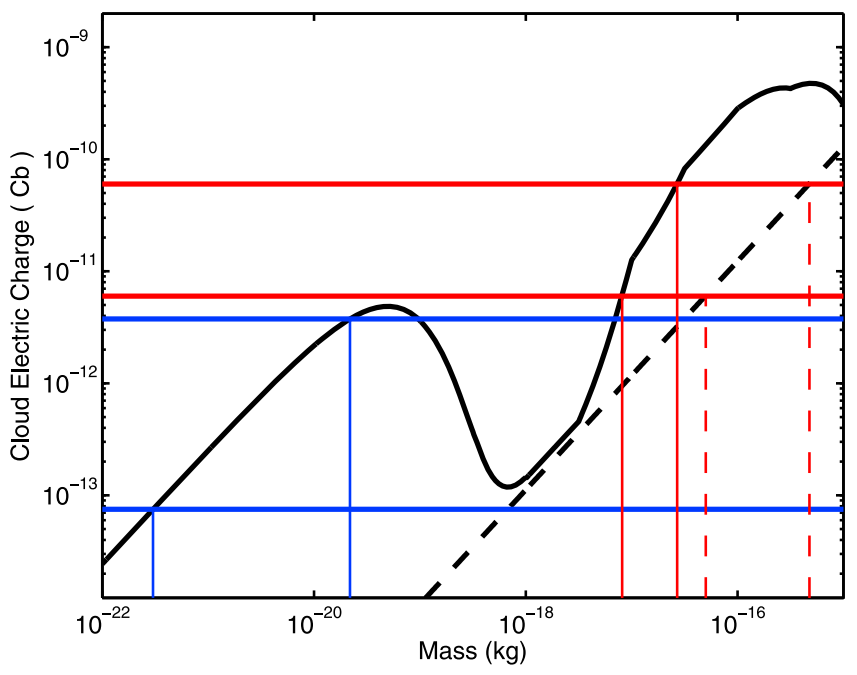

Figure 6. The charge $\mathrm{Q}$ generated by a dust impact as a function of the mass of the impinging particle is plotted in black (see the text for the assumed velocity model). The dashed line shows the charge generated by an interstellar dust impact when the spacecraft longitude is equal to the interstellar dust upstream or downstream longitude (see section 3.1). The horizontal red and blue lines present the TDS detection range when the signal is generated by the cloud's electrons recollection (calculated from equation (2), red) and through electric potential perturbation of a single antenna (equation (4), blue). The vertical lines show the grain mass range scanned when looking at triple (red) and single (blue) hits. The considered voltage detection interval is $15-150 \mathrm{mV}$ for the red lines and $3-150 \mathrm{mV}$ for the blue lines. 

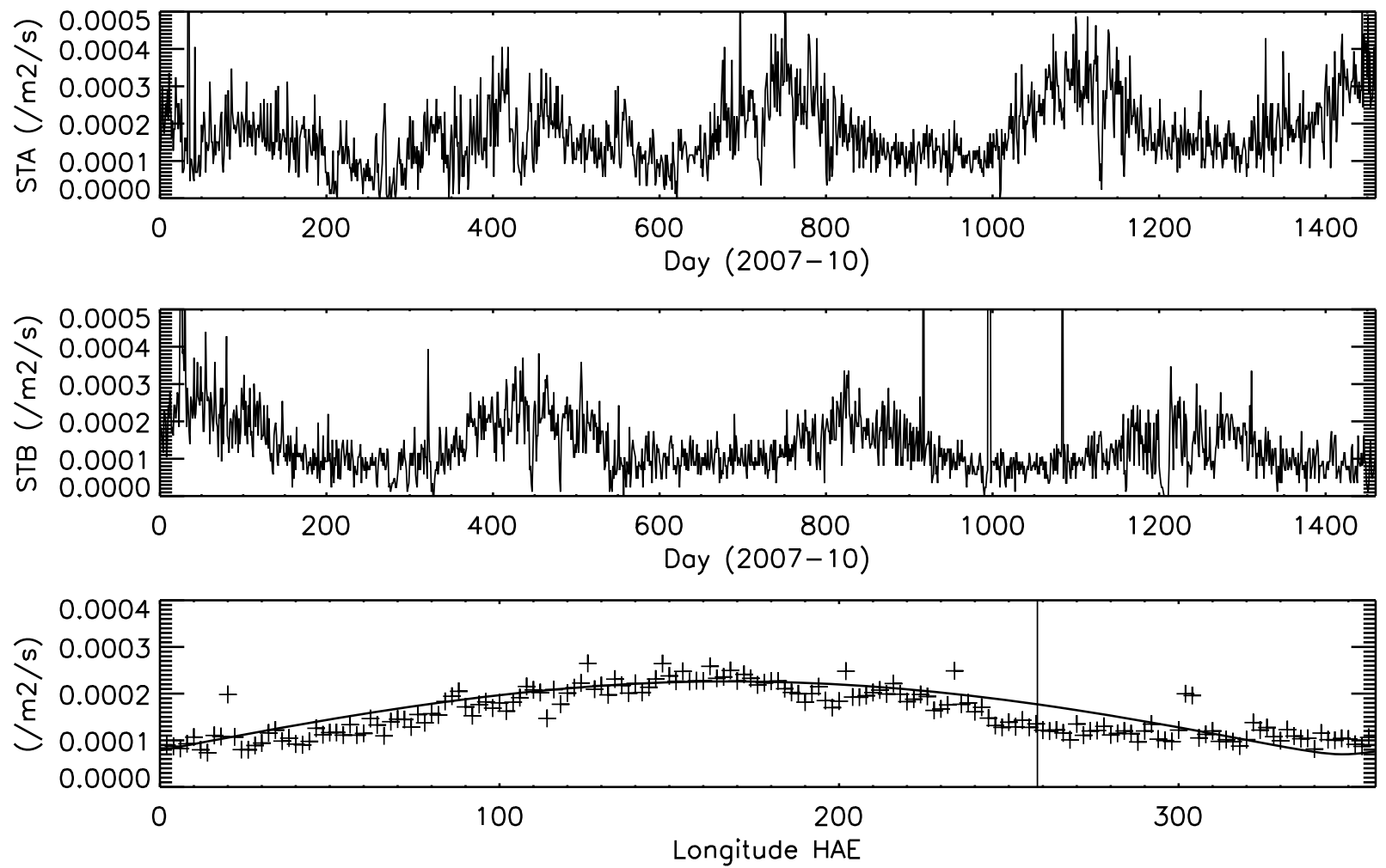

Figure 7. Flux calculated each day according to formula (5) for STEREO A and B. The observed fluxes present a modulation depending on the solar longitude. The bottom panel presents the averaged flux measured by both spacecraft as a function of the solar ecliptic longitude (the average is performed on 2 deg bins). The vertical line shows the upstream direction of the interstellar dust flow, and the best fit if the data using equation (6) is over-plotted (parameters: $\mathrm{V}_{I S D}=26 \mathrm{~km} / \mathrm{s}, V_{\beta}=80 \mathrm{~km} / \mathrm{s}, F_{\beta} \times S /\left[1 \mathrm{~m}^{2}\right]=6 \times 10^{-5} / \mathrm{m}^{2} / \mathrm{s}$, $\left.F_{I S D} \times S /\left[1 \mathrm{~m}^{2}\right]=8 \times 10^{-5} / \mathrm{m}^{2} / \mathrm{s}, \theta_{I S D}=258 \mathrm{deg}\right)$.

and high velocities (M. Horanyi, private communication, 2011), including the Cassini cosmic dust analyzer, that nevertheless detected nano-dust streams ejected by outer planets [Hsu et al., 2012].

[24] In order to link the charge $Q$ generated by an impact to the mass of the impinging dust particle, we have to assume a relationship between the mass of a dust grain and its typical velocity at 1 A.U. For this study, we use a velocity model obtained by interpolating the results from numerical simulations presented in Mann et al. [2010, Figure 5]. Roughly, nano-particles with $m<10^{-19} \mathrm{~kg}$ travel at the solar wind velocity $\sim 350 \mathrm{~km} / \mathrm{s}$, consistent with the pick-up ion like acceleration mechanism described by Czechowski and Mann [2010]. Particles in the range $10^{-18}-10^{-16} \mathrm{~kg}$ travel at velocities $\sim 50-80 \mathrm{~km} / \mathrm{s}$ as the Sun's radiation pressure accelerates these so-called $\beta$-meteoroids to such velocities at 1 A.U. This velocity range assumes that the majority of the $\beta$-meteoroids are generated within 0.3 A.U. from the Sun and that the $\beta$ parameter (equal to the ratio of the radiation pressure force to the gravitational force exerted on a particle) follows the values calculated by Wilck and Mann [1996] for interplanetary dust properties. We consider independently the interstellar dust population, assuming a $26 \mathrm{~km} / \mathrm{s}$ velocity for all particles regardless of their mass (see section 3.1). It must be noted that the velocities considered here are typical velocities, and that each of these dust populations actually have a broad velocity distribution at 1 A.U. The dust mass intervals derived hereafter are therefore characteristic of typical impacts.

[25] We calculate $Q(m)$ using equation (1) assuming an impact speed $v=\sqrt{v_{d u s t}^{2}+v_{s c}^{2}}$, where $v_{d u s t}$ is obtained from the model and $v_{s c}=30 \mathrm{~km} / \mathrm{s}$ is the STEREO spacecraft velocity. This formula for the impact speeds assumes a radial velocity for the nano-particles and $\beta$-meteoroids. For interstellar dust, this formula provides the impact speed when the spacecraft longitude is equal to the upstream or downstream interstellar dust longitude. The obtained $Q(\mathrm{~m})$ functions are plotted in Figure 6.

[26] There are several different ways for the electric charge generated by a dust impact to induce a measurable electrostatic potential difference between the spacecraft main body and at least one of the antennas [Oberc, 1996]. (1) The potential of the satellite's body drops (with respect to the antennas) through recollection of cloud electrons, (2) The potential of one of the antennas drops (with respect to the main body) through recollection of cloud electrons, (3) One of the antennas is partially enveloped by the expanding cloud and directly affected by the cloud's electrostatic field (see Appendix A). Scenarios (2) and (3) are very dependent on the impact geometry and are likely responsible for pulses detected on a single antenna. Scenario (1) does not favor a particular 
antenna and should generate pulses of the same order of magnitude on the three antennas, rather independently of the impact localization. It is the likely scenario for the triple-hits. Scenario (2) generates negative voltage pulses, which are rare and will not be discussed further in this work.

\subsubsection{Triple-Hits: $\boldsymbol{\beta}$-Meteoroids and Interstellar Grains}

[27] The detection of the spacecraft charging after a dust impact has already been used as the basis for dust detection by Voyager [e.g., Gurnett et al., 1991; Meyer-Vernet et al., 1996, 1998; Oberc, 1994] and Cassini [Meyer-Vernet et al., $2009 \mathrm{c}]$. The signal in this case is generated by the recollection of the cloud's electrons by the positively charged spacecraft surface. The potential of the spacecraft then undergoes a drop of the order $\delta V_{s c} \simeq-Q / C_{s c}$, where $Q$ is given by (1) and $C_{s c}$ is the spacecraft body capacitance (for STEREO we use the capacitance of a perfectly conducting rectangular cuboid of dimensions $2.3 \mathrm{~m} \times 1.2 \mathrm{~m} \times 1.3 \mathrm{~m}$, that is about $200 p F$ ). The signal detected by a monopole is $\delta V=V_{\text {ant }}-V_{s c}$. As the potential of the antennas is roughly constant on the $\sim$ ms timescale of the impact (small variations might happen as the antennas can recollect part of the cloud's electrons), the three monopoles will detect a voltage pulse of the same order of magnitude

$$
\delta V_{3 h i t s} \simeq \Gamma Q / C_{s c},
$$

where $\Gamma \sim 0.5$ is the gain factor of the antennas due to their capacitive coupling with the base [Bale et al., 2008]. The values of the charges that can be detected by the receiver for voltage pulses in the range $15 \mathrm{mV}<\delta V_{3 \text { hits }}<150 \mathrm{mV}$ are shown as red horizontal lines in Figure 6. The empirical choice of the $15 \mathrm{mV}$ lower bound is due to the use of the TDSmax to analyze these signals. Indeed, as already discussed, the TDSmax mode does not enable us to verify dustspike shape of the waveform. Thus we did not consider amplitudes smaller than $15 \mathrm{mV}$, for which a similar maximum on the three antennas could be generated by strong solar wind density fluctuations, or less frequently by intense Langmuir wave packets.

[28] It can be seen in Figure 6 that the mass scanned depends on the velocity of the dust population considered, which we cannot measure independently. Thus two groups of particles mainly cause the triple hits: the $\beta$-meteoroids, with radial velocities of the order of $50-80 \mathrm{~km} / \mathrm{s}$, and interstellar dust particles, with velocities of the order of $26 \mathrm{~km} / \mathrm{s}$. For $\beta$-particles the triple hit signals correspond to grains in the mass range $8 \times 10^{-18}-3 \times 10^{-17} \mathrm{~kg}$, or equivalently to sizes $r_{d} \sim 90 \mathrm{~nm}-0.14 \mu \mathrm{m}$ (assuming a spherical grain of radius $r_{d}$ and mass density $\rho=2.5 \times$ $10^{3} \mathrm{~kg} / \mathrm{m}^{3}$ ). For interstellar dust particles, the mass range is $5 \times 10^{-17}-5 \times 10^{-16} \mathrm{~kg}$, or $r_{d} \sim 0.17-0.36 \mu \mathrm{m}$.

[29] It must be pointed out that a fraction of the triple hits is generated by particles orbiting at 1 A.U. at Keplerian velocities. Such particles impact the spacecraft with an average $20 \mathrm{~km} / \mathrm{s}$ velocity, and thus, according to equations (1) and (2), have a mass $\sim 5 \times 10^{-16}-5 \times 10^{-15} \mathrm{~kg}$. Since the cumulative mass flux decreases with the mass, this component should be small compared to the $\beta$-meteoroids component, and can be separated from the interstellar one using the longitudinal modulation of the latter (see section 3.1).

\subsubsection{Single-Hits: Fast Nanometer Grains}

[30] When an impact occurs close enough to an antenna, the direct detection of the plasma cloud is made possible through the perturbation of the electrostatic equilibrium around the antenna. Close enough here means that the impact occurred at a distance smaller than the maximum radius $R_{C}$ where the plasma cloud can be differentiated from the background plasma:

$$
R_{C} \sim\left(3 Q / 4 \pi e n_{a}\right)^{1 / 3},
$$

$n_{a}$ being the number density of the ambient solar wind electrons, typically $\sim 5 \mathrm{~cm}^{-3}$ at 1 A.U.

[31] In this case, the voltage will be larger on the antenna close to the impact, but the evaluation of its dependence on the charge $Q$ has not been experimentally performed yet. Under the presumably realistic assumption that the plasma cloud expansion is collisionless, F. Pantellini et al. (Nano dust impacts on spacecraft and boom antenna charging, submitted to Annales Geophysicae, 2012) recently showed that the cloud is quasi-neutral (that is, the plasma Debye radius is smaller than the cloud radius) and that its electric field is too weak to be measured in the simplest case of a plasma cloud expanding isotropically in vacuum and subjected to its proper electric field only.

[32] The antenna potential variation produced by the impact of a fast nano-dust is also much greater than the Coulomb field of the charge $Q$. This feature can be explained by considering the dynamic equilibrium of the photoelectrons and ambient solar wind electrons currents which determine the floating potential of a radio antenna in the interplanetary medium. This equilibrium can be described as follows [Meyer-Vernet, 2007] : the antenna, under solar UV illumination, emits a photoelectron current $I_{p h} \sim 50 \mu \mathrm{A} / \mathrm{m}^{2}$ (at 1 A.U.). It also collects electrons from the ambient solar wind, at a much slower rate $I_{s w} \sim 1-$ $2 \mu \mathrm{A} / \mathrm{m}^{2}$ (at 1 A.U.). The antenna thus charges positively until it reaches a large enough potential to trap an important fraction of the photoelectrons, so that the current of the escaping photoelectrons equalizes the current of the incoming solar wind electrons.

[33] The large value of $I_{p h}$ compared to $I_{s w}$ makes that a transient perturbation of the photoelectrons dynamics can readily change the antenna potential. Under the plausible assumption (cf. section A2) that the presence of the plasma cloud modifies the trajectories of the trapped photoelectrons so that an important fraction of them do not return to the antenna, we derive, in Appendix A, a calibration formula for the single hits. It links the amplitude of the voltage pulse $\delta V_{1 \text { hit }}$ to the charge $Q$ of the impact:

$$
\delta V_{1 h i t} \sim \Gamma\left(\frac{T}{2 \pi e n_{a} L R_{s c}^{2}}\right) Q
$$

where $R_{s c} \sim 1 \mathrm{~m}$ is of the order of magnitude of the spacecraft size, and $L$ is the antenna length. $T$, of the order of the photoelectron temperature (in $\mathrm{eV}$ ), is precisely defined in section A1. Equation (4), combined with the assumption that the small signals observed on the two "non-hit" antennas are due to the electron recollection by the spacecraft surface, and thus related to $Q$ through equation (2), enables us to reproduce the antenna voltage correlations appearing in the data 
(Figures 4 and 5), as shown in section A3. This strongly supports the proposed physical mechanism, and makes very unlikely the signals to be generated by any kind of noise.

[34] Using the $V_{1 \text { hit }}$ to $Q$ relation (4) with the parameters $T=3 \mathrm{eV}, n_{a}=5 \mathrm{~cm}^{-3}, L=6 \mathrm{~m}$ and $R_{s c}=1 \mathrm{~m}^{2}$, and the charge to mass relationship (1), one can evaluate the mass range scanned by the single-hits as $3 \times 10^{-22}-2 \times 10^{-17} \mathrm{~kg}$. This large band is due to the fact that, as seen in Figure 6, the function $m(Q)$ is multivalued in the $Q$ range $10^{-12}$ $10^{-13} \mathrm{C}$, so that a $150 \mathrm{mV}$ pulse could be generated by a $\sim 2 \times 10^{-17} \mathrm{~kg}$ grain as well as a $\sim 2 \times 10^{-20} \mathrm{~kg}$ grain. However, since the dust cumulative mass flux decreases steeply with mass in this mass range [Grün et al., 1985], $150 \mathrm{mV}$ impacts will most likely be caused by $\sim 2 \times 10^{-20} \mathrm{~kg}$ grains. The effective mass range scanned when analyzing the single-hits between 3 and $150 \mathrm{mV}$ is therefore $3 \times 10^{-22}-2 \times 10^{-20} \mathrm{~kg}$, corresponding to sizes $\sim 3-12 \mathrm{~nm}$.

\section{Fluxes of Nano and Sub-micron Dust at 1 A.U. Detected by STEREO}

[35] In this section, we present the fluxes detected by the STEREO spacecraft for dust grains in the $0.1-0.3 \mu \mathrm{m}$ and 3-12 nm ranges, during the period 2007-2010.

\section{1. $\beta$-Meteoroids and Interstellar Dust Fluxes at 1 A.U.}

[36] We used the TDSmax data set to calculate the fluxes day by day of particles in the range $0.1-0.3 \mu \mathrm{m}$. The use of an instrument providing a single measurement per minute is justified by the obtained result of an impact rate $\sim 10 /$ day, which makes the occurrence of a double impact within the same minute extremely unlikely (according to Poisson statistics, this probability is $\sim 10^{-5}$ ).

[37] Dust particles in this range are detected when impacting the spacecraft body. The number of dust hits per second on the spacecraft is $N_{s}=S F\left(m_{\min }, m_{\max }\right)$, where $S$ is the cross-sectional surface of the spacecraft $\left(S \sim 1-3 \mathrm{~m}^{2}\right.$ for STEREO's cuboid body) and $F\left(m_{\min }, m_{\max }\right)$ the flux of particles having a mass between $m_{\min }$ and $m_{\max }$. Here the observed mass interval is defined by the voltage interval considered in the data (section 2.4.2), and the flux in $\mathrm{m}^{-2} \mathrm{~s}^{-1}$ is simply

$$
F\left(m_{\min }, m_{\max }\right) \simeq N_{d a y} /(24 \times 3600 \times S),
$$

where $N_{\text {day }}$ is the number of triple-hit recorded during a given day. Figure 7 presents the results of the dust flux (5) measured by the TDSmax for each day of the period 2007-2010. This figure shows that, in addition to a constant level of flux, there is an additional flux component varying with the solar longitude (the time evolution of longitude is different for the two spacecraft: STEREO A travels faster than the Earth, STEREO B slower).

[38] This component is consistent with being produced by dust from interstellar origin (ISD). ISD flows with respect to the Sun along the $\theta_{I S D} \sim 259 \mathrm{deg}$ ecliptic longitude with a speed $V_{I S D} \sim 26 \mathrm{~km} / \mathrm{s}$ [Mann, 2010], the drift velocity of the solar system with respect to the interstellar medium that surrounds it. Taking into account that the measured flux is equal to $F=N \times V$ where $N$ is the dust number density and $V$ the relative velocity of the spacecraft with respectto the dust, the total flux detected by STEREO is derived from the variation of $V$ with the longitude $\theta$, and is equal to

$$
F=F_{\beta} \sqrt{1+\left(\frac{V_{S C}}{V_{\beta}}\right)^{2}}+F_{I S D} \sqrt{1+\left(\frac{V_{S C}}{V_{I S D}}\right)^{2}-2 \frac{V_{S C}}{V_{I S D}} \sin \left(\theta-\theta_{I S D}\right)} .
$$

Here $V_{S C} \simeq 30 \mathrm{~km} / \mathrm{s}$ is the orbital speed of the STEREO spacecraft. In order to estimate the uncertainties, we varied the parameters in the following range $V_{\beta} \simeq 50-80 \mathrm{~km} / \mathrm{s}$ for the radial velocity of the $\beta$-meteoroids population, $V_{I S D} \simeq 20-35 \mathrm{~km} / \mathrm{s}$ for the interstellar dust velocity, and $S=1-3 \mathrm{~m}^{2}$ for the spacecraft cross-sectional surface. Using these parameters and fitting the curve presented in Figure 7 with a least squares method, we derive the fluxes: $F_{\beta} \simeq 1-6 \times 10^{-5} / \mathrm{m}^{2} / \mathrm{s}, F_{I S D} \simeq 2-10 \times 10^{-5} / \mathrm{m}^{2} / \mathrm{s}$, the uncertainty being dominated by the value of the collecting surface $S$ assumed. The upstream ecliptic longitude of the ISD obtained through the fit is $\theta_{I S D} \simeq 258 \mathrm{deg}$, within a degree of the expected value.

[39] Interstellar dust with mass $>3 \times 10^{-16} \mathrm{~kg}$ enters the solar system without significant deflection. Within the solar system the flux is modified by focusing or defocusing magnetic field configurations that vary with solar cycle and by gravitational focusing, so that the interstellar dust flux near Earth orbit is neither constant in time nor in longitude [Mann, 2010]. However, these STEREO measurements are in a good agreement with the results obtained by the Ulysses spacecraft [Krüger et al., 2007], with measured fluxes during the period 1992-2006 ranging from $5 \times 10^{-5}$ to $4 \times 10^{-4} /$ $\mathrm{m}^{2} / \mathrm{s}$ in a somewhat larger mass range. The STEREO fluxes also overlap with the fluxes derived from the Cassini spacecraft measurements performed between 0.7 and $1.2 \mathrm{~A}$. U., that report a mean ISD flux of $2.5 \pm 0.5 \times 10^{-5} \mathrm{~m}^{-2} \mathrm{~s}^{-1}$ in the mass range $5 \times 10^{-17}-10^{-15} \mathrm{~kg}$ [Altobelli et al., 2003]. Given the differences between the Ulysses and the Cassini measurements as well as the flux variations predicted by theory we conclude that our result agrees with the other observations.

[40] To conclude this part, let us note that equation (6) neglects some effects, such as the velocity dispersion of the dust particles, as well as the fact that the mass range scanned varies with the relative velocity of the impact, and thus, for the ISD, with the spacecraft longitude. Such effects will be investigated in a forthcoming article specifically dedicated to the interstellar dust observation. However, the present data and ongoing measurements provide an excellent data set for detailed studies of the ISD flux near 1 A.U.

\subsection{Nanometer Dust at 1 A.U.}

\subsubsection{Measurement of the Flux}

[41] The flux for the nanometer sized particles responsible for the single-hit events is less straightforward to determine. Indeed, the large number of hits per minute prevents us from using the TDSmax data. We instead used the individual event data, counting for each day the total number of singlehits detected by the instrument in the range $3 \mathrm{mV}-150 \mathrm{mV}$, and dividing by the total instrument sampling time during this day to obtain a number of impacts per second. This method would be valid if the events where randomly chosen 

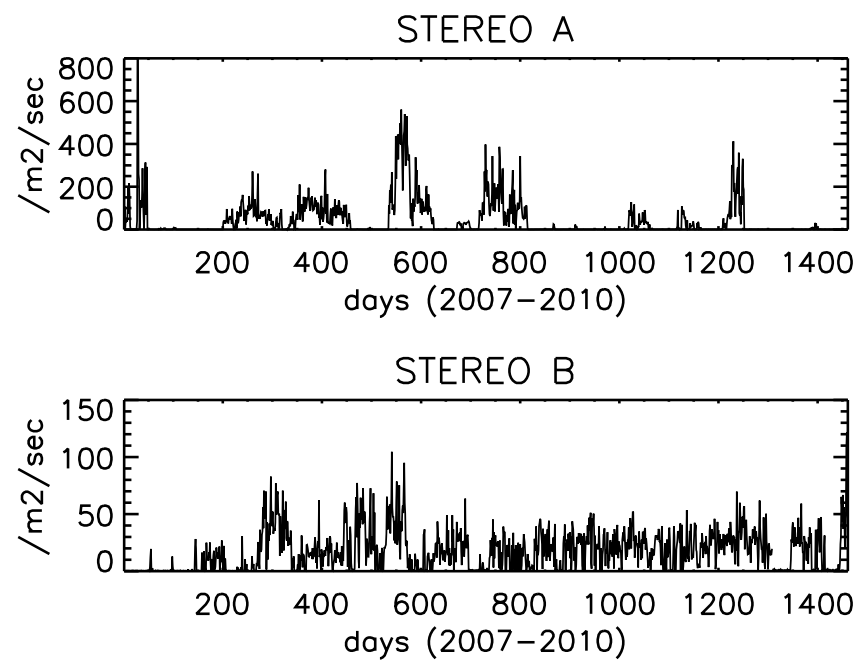

Figure 8. Flux $F\left(m_{\min }\right)-F\left(m_{\max }\right)$ in the mass range $3 \times 10^{-22}-2 \times 10^{-20} \mathrm{~kg}$, for nano-dust detected through single hits impacts on the $\mathrm{X}$ (or Z) monopole of STEREO A (or $\mathrm{B}$, respectively), during each day of the period 2007-2010. The points are obtained analyzing the TDS event data, and thus provide an overestimation of the actual flux (see the text). The fluxes are calculated according to equation (7). On STEREO A, the nano-dust appears in bursts, in contrast to the more constant flux observed on STEREO B. The nano-dust is seen at higher rates by STEREO A.

within the day, or if the flux was constant over the day. Unfortunately, the flux does generally vary in a day, and the event selection criterium is likely to favor events occurring during high flux periods. Thus the fluxes given here are overestimated by a factor difficult to precisely evaluate (see the discussion in sections 3.3 and 4). Nevertheless the relative variations of the flux provided by this method should be reliable, as the event selection method does not vary in time.

[42] The single hits are detected if they impact the spacecraft at a distance smaller than $R_{C}$ (3) from an antenna. Assuming that the cumulative flux distribution varies as $F=F_{0} m^{-\delta}$, with $\delta=5 / 6$ [Dohnanyi, 1969], the flux can be estimated as a function of the number $N$ of impacts per seconds as

$$
N=\int_{m_{\min }}^{m_{\max }} R_{C}^{2} \delta F_{0} m^{-(\delta+1)} d m
$$

where $R_{C}$ depends on $m$ through the charge $\mathrm{Q}$ as given by (3). In the considered mass range one can assume that $Q$ varies linearly with $m$, and that $v \sim 350 \mathrm{~km} / \mathrm{s}$ (Figure 6). Thus using (1) and (3) one obtains $R_{C} \sim 5.5 \times 10^{6} \mathrm{~m}^{1 / 3}$, and thus $N \sim 1.5 \times 10^{14}\left(m_{\min }^{-1 / 6}-m_{\max }^{-1 / 6}\right) F_{0}$. Figure 8 shows the flux calculated according to (7) with values of $m_{\min }$ and $m_{\text {max }}$ provided in section 2.3 .2 , and $N$ derived by counting the pulses detected in the TDS events. One can see that the flux is extremely variable in time (from $F \simeq 0$ to $600 / \mathrm{m}^{2} / \mathrm{s}$ ), and is different on the two spacecraft. This last point raises the question of the importance of the antenna-spacecraft geometry in the detection of the nano-dust, as their detection through perturbation of the antenna photoelectron sheath strongly depends, as already mentioned, on the location of the dust impact with respect to the antenna.

[43] The 4-years averaged values of the fluxes on STEREO A and $\mathrm{B}$ are respectively $F_{A} \simeq 44 / \mathrm{m}^{2} / \mathrm{s}$ and $F_{B} \simeq 17 / \mathrm{m}^{2} / \mathrm{s}$, which is more than an order of magnitude above the value inferred from the Grün et al. [1985] model (see Figure 10).

\subsubsection{Comparison With Previous Studies}

[44] The interplanetary nanometer sized dust has previously, and for the first time, been observed by Meyer-Vernet et al. [2009a]. In that article, the authors used the data from the S/WAVES Low Frequency Receiver (LFR) during the year 2007 to analyze the dust impacting the spacecraft with a few seconds resolution (thus involving an average on the dust impacts during the integration time of the instrument). Figure 9 shows a remarkably good agreement between the flux variations provided by the two instruments. This ensures that the origin of the signal detected by the LFR is effectively nano dust impacts (this had to be assumed in the original work, as the waveforms had not been analyzed in detail). It also shows that both LFR and TDS instruments can be used independently to study the flux variation of the nano dust. Nevertheless, the small flux of the sub- $\mu \mathrm{m}$ sized dust makes it impossible to use LFR for the larger grains.

\subsection{Comparison of the Dust Fluxes Derived From S/WAVES With an Interplanetary Dust Flux Model}

[45] Figure 10 summarizes the fluxes obtained from the analysis of the S/WAVES data during the period 20072010, and compares them to the classical Grün et al. [1985] dust flux model. A first look shows a good agreement between the STEREO observations and the model. Points and error bars are obtained in the following way. For each point we center the mass interval on the smallest mass $m_{\text {min }}$ determined in section 3. Then, we assume an order of magnitude error on the mass as a consequence of the uncertainties both on the expression (1) for the charge and on the velocity of the impinging grains. The fluxes obtained in the previous section are not the cumulative flux $F\left(m_{\min }\right)$ for the minimum mass, but the difference $F\left(m_{\min }\right)-$ $F\left(m_{\max }\right)$. In order to obtain $F\left(m_{\min }\right)$, we make the assumption that the flux dependence on the mass is $F=F_{0} \times m^{-\delta}$. Then $F\left(m_{\min }\right)$ is given by

$$
F\left(m_{\min }\right)=F_{\text {measured }} \times \frac{m_{\min }^{-\delta}}{m_{\min }^{-\delta}-m_{\max }^{-\delta}},
$$

where $F_{\text {measured }}$ is the result presented in the previous section in a given mass range $m_{\min }-m_{\max }$. Following Grün et al. [1985], we used $\delta \sim 1 / 2$ in the sub-micron size range and $\delta \sim 5 / 6$ in the nanometer size range.

[46] The red cross shows the upper and lower values of the obtained cumulative flux of $\beta$-meteoroids. The flux lies slightly below the interplanetary dust model curve. As pointed out in section 2.4.2, a part of the flux $F_{\beta}$ is due to particles on Keplerian orbits. Assuming a cumulative mass flux decreasing with a $\delta \sim 1 / 2$ power law, one finds this proportion to be $\sim 10 \%$.

[47] The green cross shows the interstellar dust measurement which, as already pointed out in section 3.1 , is in good agreement with previous observations. 


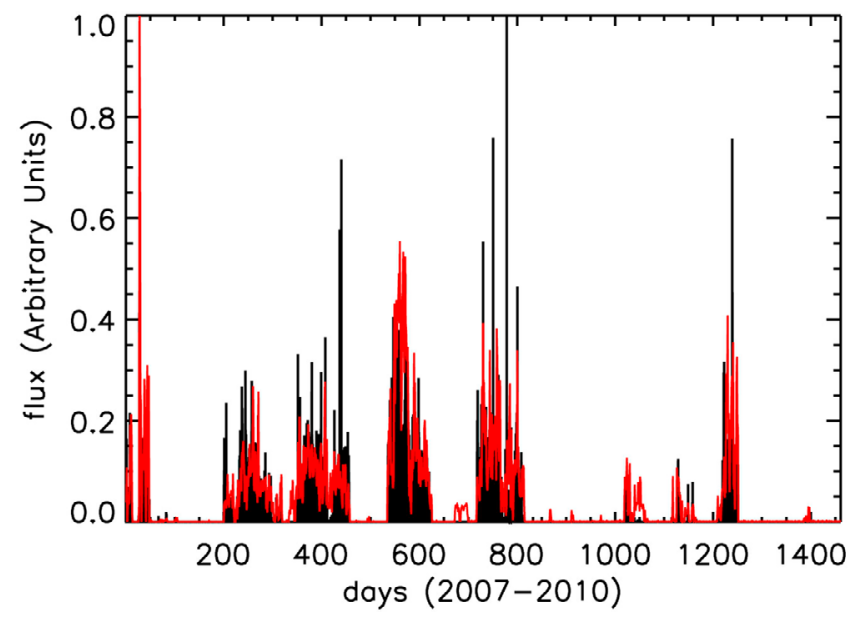

Figure 9. Comparison of the fluxes obtained during the period 2007-2010 by the LFR analysis (black curve) and the TDS Events analysis (red curve) on STEREO A. Fluxes are in arbitrary units. The agreement between the two curves is very good, showing that the two instruments each enable an accurate measurement of the nano dust flux variation.

[48] The upper limit of the blue cross shows the 4-year averaged cumulative flux $F\left(m_{\min }=3 \times 10^{-22} \mathrm{~kg}\right)$. As previously mentioned, this nano-dust flux has been measured by counting day by day the number of peak-shaped (singlehits) signals in theTDS events recorded during this day, and by dividing this number by the total TDS event time $T_{t d s}$ in the day. Usually $T_{t d s} \sim 5 \mathrm{~s}$, so that, in the worst case where there would be dust only during the events, the flux would be overestimated by a factor $24 \times 3600 / T_{t d s} \sim 10^{4}$ (lower limit of the blue cross). The real factor is probably much smaller than this, but cannot be determined from the present data. The results obtained in the nano-dust range, both through TDS and LFR analysis, are the first observation of interplanetary dust flux at 1 A.U. for mass below $10^{-21} \mathrm{~kg}$. They are compatible with the Grün et al. [1985] model, and allow for further studies of the dust flux models in this mass range.

\section{Discussion and Conclusion}

[49] We have shown in this article that the S/WAVES instrument on STEREO can be used as a dust detector with convincing results both in the nanometer and sub-micrometer size ranges. In the latter case, the orbital motion of the spacecraft allows us to distinguish between interstellar and interplanetary dust components. Our results reasonably agree with previous studies and with current dust flux models. We also propose a physical mechanism (see section 2.4.3 and Appendix A) for the generation of the single-hits which does remarkably well in explaining the TDS observations. Nevertheless, questions remain about the difference of averaged nano-dust fluxes observed on the two spacecraft (a factor $\sim 2.5$ between them), on the difference of the time variations of these fluxes (the flux is more bursty on STEREO A), as well as about the reason why the monopole $\mathrm{X}(\mathrm{Z})$ is preferentially hit by nano-dust on STEREO A ( $\mathrm{B}$, respectively). The answers to these questions could depend both on the particular geometry of these spacecraft and on the directionality of the nano-dust streams due to the interplanetary magnetic field driven dynamics of these small grains. Differences between the material of the two spacecraft thermal blankets could also explain differences in the observed fluxes.

[50] Thus the detection and flux measurements of dust particles with radio antennas provide an interesting option to complement traditional dust analyzers for future heliosphere exploration missions. To optimize these measurements a few instrumental developments could be (or are already) considered: an automatized dust counter registering the amplitude and time of the impacts (or equivalently an unbiased TDS mode) would greatly reduce the error bars on the flux measurements especially in the nano-size range where the fluxes are high. A larger dynamical range (S/WAVES TDS saturates at $\sim 170 \mathrm{mV}$ ) would enable to scan larger (and different) mass ranges. A simple antenna geometrical configuration (with well separated antennas) would allow gathering more information on the single-hits, in particular concerning the dust grain velocity vector. Ground based experiments could be realized to check the validity of equation (1) in larger mass and velocity ranges, in particular for nano-particles traveling close to $300 \mathrm{~km} / \mathrm{s}$. This technique could be a powerful observational capability for the upcoming NASA Solar Probe Plus to the solar corona, the FIELDS experiment of which includes four electric field antennas. Other proposed radio concepts in space, such as lunar based large area radio arrays could consider already dust as a science goal in itself.

[51] The detection method and the results presented in this article constitute a first step in the use of the TDS data in

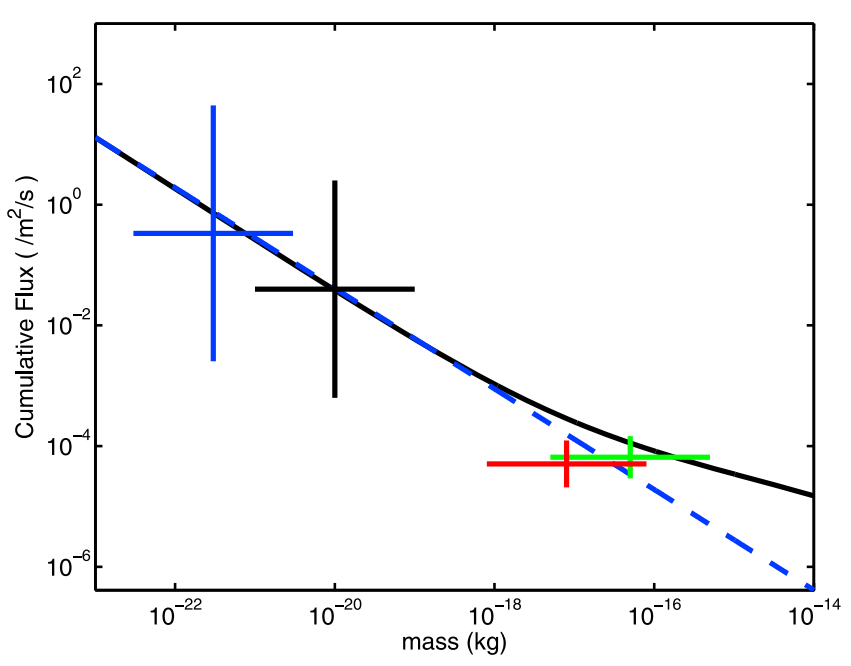

Figure 10. Comparison of the flux measurements performed in this article using the S/WAVES TDS, and a classical dust flux model [Grün et al., 1985] given by the solid curve. The dashed curve is the extrapolation of the model with a $m^{-5 / 6}$ power law, from the small bodies fragmentation theory [Dohnanyi, 1969]. The red cross shows the $\sim 0.1 \mu \mathrm{m}$ flux measurements. The green cross shows the result derived from the $0.1-0.3 \mu \mathrm{m}$ interstellar dust measurement. The blue cross shows the 3-12 nm measurements (4-year average). The black cross shows the LFR result of Meyer-Vernet et al. [2009a]. The results presented are the derived cumulative fluxes $F\left(m_{\min }\right)$ (see the text for the details). 
dust detection, and will provide a basis for numerous future works. The interstellar dust data set here obtained will be the purpose of a forthcoming article, where the time variation of their flux and direction with respect to the Sun will be investigated. Numerical simulations and theoretical studies could be performed in order to improve our understanding of the electric signal generation, especially for the single hits. The detection capability could also be improved by investigating whether the dust impact location can be determined from our data. The study of the negative hits distribution could be part of such a study. Forthcoming works also include the study of another TDS data set, the Langmuir Wave Statistics (LWS), that provides histograms of the electric potential amplitude collected on a minute timescale. This data set clearly contains relevant information on the dust fluxes and mass distribution. Finally, the presented technique could be applied to spacecraft presently providing waveforms data sets, such as Cassini, WIND, etc.

\section{Appendix A: Detection Mechanism for the Single-Hits}

\section{A1. Signal Generated by a Local Perturbation of the Photoelectron Current Around an Antenna}

[52] The typical photoelectron current emitted by a spaceborne antenna at 1 A.U. is $I_{p h} \sim 50 \mu \mathrm{A} / \mathrm{m}^{2}$ [see, e.g., Cully et al., 2007 and references therein]. Therefore, if the return photoelectron current is suppressed on a length $l$ of the antenna, during a time $\tau$, the antenna charge will increase by

$$
\delta q \sim I_{p h} \times(2 a l) \times \tau,
$$

where $a$ is the radius of the antenna. The voltage increase induced by the charging of the antenna is $\delta V=\delta q / C_{a}$ where $C_{a}$ is the capacitance of the antenna in the low frequency limit [Meyer-Vernet and Perche, 1989],

$$
C_{a}=\frac{2 \pi \epsilon_{0} L}{\ln \left(\lambda_{d} / a\right)}
$$

( $\lambda_{d}$ is the plasma Debye length, $L$ the length of the antenna boom and $\epsilon_{0}$ the vacuum permittivity). The receiver connected to the monopole will thus measure a voltage pulse of amplitude

$$
\delta V \sim \Gamma\left[\frac{I_{p h} a \tau \ln \left(\lambda_{d} / a\right)}{\pi \epsilon_{0}}\right] \frac{l}{L},
$$

with $\Gamma$ the gain of the monopole. The time $\tau$ of the perturbation can be empirically determined from the observation of the typical risetime of the signals, $\tau \sim 10-20 \mu \mathrm{s}$. Using this value and the S/WAVES antenna parameters $L=6$ mand $a=1.6 \mathrm{~cm}$ close to the cylindrical antenna base [Bale et al., 2008], the factor between brackets in (equation A3) can be evaluated around $\sim 2-4 \mathrm{~V}$. We shall refer to this factor as to an effective temperature $T \sim 2-4 \mathrm{eV}$, of the order of the photoelectron temperature.

\section{A2. Calculation of the Average Signal}

[53] Here, we shall assume that the plasma cloud generated by a dust impact changes the photoelectrons trajectories so that the return current is canceled during a short time $\tau$, on the length $l$ of the antenna embedded in the plasma cloud.

[54] A plausible scenario for this perturbation (detailed by F. Pantellini et al. (submitted manuscript, 2012) is the following: the photoelectron trajectory is hard to modify in the radial direction (with respect to the antenna), as an energy of several volts (of the order of the antenna potential) has to be provided for the electrons to escape the antenna. Such an energy clearly cannot be provided by the dust induced plasma cloud. In contrast, because of angular momentum conservation, the tangential energy of the emitted photoelectrons decreases as the inverse square distance of the antenna axis. Hence, the photoelectron tangential energy is small, of the order of $T_{p h}\left(a / R_{p h}\right)^{2} \sim 10^{-4} \mathrm{eV}$ (where $R_{p h} \sim 1 \mathrm{~m}$ is the typical radial size of the photoelectron sheath around the antenna). The dust induced plasma electrostatic field can easily provide this small amount of energy, and thus place the photoelectrons on elliptical orbits around the antenna, resulting in a transient cancelation of the return current.

[55] Considering a dust impacting at a distance $r$ from the antenna, and a spherical geometry for the expansion of the cloud until its maximum radius $R_{C}$ (3) (i.e. the plasma cloud is a half-sphere of radius $R_{C}$ centered on the impact point), the length of antenna within the plasma cloud is

$$
l=\sqrt{R_{C}^{2}-r^{2}}
$$

We assume a cylindrical symmetry around the antenna, so that the probability that an impact occurs at a distance between $r$ and $r+d r$ from the antenna is $d p \propto 2 \pi r d r$. Using $\delta V \sim \Gamma T(l / L)$, we find that the average signal produced by a cloud of charge $Q$ is

$$
\langle\delta V\rangle_{r}=\frac{\Gamma T}{L} \int_{0}^{\min \left(R_{C}, R_{s c}\right)} \sqrt{R_{C}^{2}-r^{2}} \frac{2 r d r}{R_{s c}^{2}}
$$

the integration of which provides,

$$
\langle\delta V\rangle_{r}= \begin{cases}\frac{2 \Gamma T}{3} \frac{R_{C}^{3}}{L R_{s c}^{2}} & \text { if } R_{C}<R_{s c} \\ \frac{2 \Gamma T}{3} \frac{R_{C}^{3}}{L R_{s c}^{2}}\left(1-\left(1-R_{s c}^{2} / R_{C}^{2}\right)^{3 / 2}\right) & \text { if } R_{C}>R_{s c}\end{cases}
$$

where the length $R_{s c}$ (of the order of the spacecraft linear dimension) has been introduced to normalize the probability. Using the relationship (3), one obtains

$$
\frac{2 T}{3} \frac{R_{C}^{3}}{L R_{s c}^{2}}=\left(\frac{T}{2 \pi e n_{a} L R_{s c}^{2}}\right) Q .
$$

\section{A3. Prediction and Observation of Voltage Correlations Between the Three Monopoles}

[56] To check the validity of the proposed scenario for the generation of the single-hit pulses, we calculate here the correlations that should be observed between the voltage peaks on the different monopoles.

[57] The cloud's electrons recollection by the spacecraft body generates a pulse $\delta V \sim Q / C_{s c}$ on the three monopoles. 


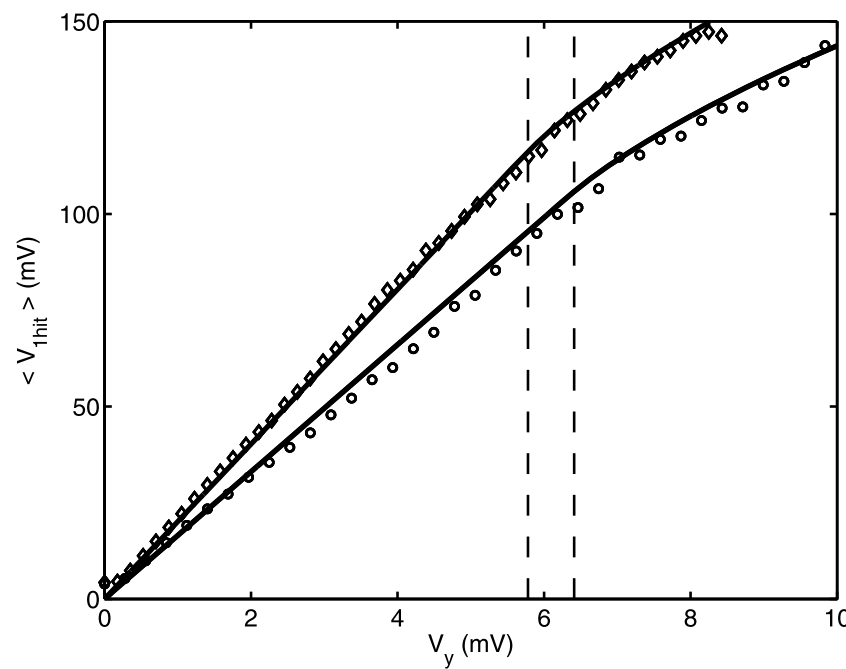

Figure A1. The average of the amplitude on the $X(Z)$ monopole on STEREO A (B, respectively) is represented by diamonds (circles, respectively) as a function of the amplitude detected on the Y monopole (which is proportional to the charge $\mathrm{Q}$ in the plasma cloud). The theoretical expression (equation A6) is over-plotted for $T=2.5 \mathrm{~V}$ (A) and $T=2.2 \mathrm{~V}(\mathrm{~B})$, the other parameters being $R_{s c}^{2}=0.7 \mathrm{~m}^{2}$ (A) and $R_{s c}^{2}=0.75 \mathrm{~m}^{2}(\mathrm{~B}), n_{a}=5 \mathrm{~cm}^{-3}$, and $L=6 \mathrm{~m}$. The vertical dashed lines show the value $V_{y}$ for which $R_{C}=R_{s c}$.

This value is small compared to the amplitude of the pulse generated by the photoelectron current perturbation, thus we cannot expect to observe it on the monopole embedded in the plasma cloud. Nevertheless, the two other monopole should present a voltage signal of this order of magnitude.

[58] For instance, if the $X$ monopole is embedded in a plasma cloud of charge $Q$, one should expect $\delta V_{x} \sim \Gamma T Q /$ $\left(2 \pi e n_{a} L R_{s c}^{2}\right)$, and $\delta V_{y, z} \sim \Gamma Q / C_{s c}$. Thus the ratio $\delta V_{x} / \delta V_{y, z}$ should be independent of $Q$. Using the parameters : $n_{a}=$ $5 \mathrm{~cm}^{-3}$ (solar wind density at 1 A.U.), $T=2-4 \mathrm{eV}$ (cf. A1), $L=6 \mathrm{~m}$ and $R_{s c}^{2}=1 \mathrm{~m}^{2}$ (size of STEREO's antennas and body), the ratio can be numerically evaluated as $\delta V_{x} / \delta V_{y, z} \sim 13-26$. The prediction that the ratio should not depend on $Q$, as well as its numerical estimate, are fully supported by the results presented in Figures 4 and 5 .

[59] To go further, we calculated, using the TDS event data set, the average value $\left\langle V_{1 h i t}\right\rangle$ as a function of $V_{y}$, on both STEREO A and B spacecraft, and compared it with the theoretical results provided by the formulas (A6) (for $\left.\left\langle V_{1 h i t}\right\rangle\right)$ and (2) (for $V_{y}$ ). The results (presented in Figure A1) show a striking agreement between the predictions and the observations, for realistic values $T \sim 2.5 \mathrm{eV}$ and $R_{s c} \sim 0.85 \mathrm{~m}$.

[60] The small difference between the inferred values of $R_{s c}$ on STEREO A and $\mathrm{B}$ is probably due to geometrical effects while the small difference between the cloud temperature on the two spacecraft is likely due to differences in the antenna gain ratios $\Gamma_{X} / \Gamma_{Y}$ and $\Gamma_{Z} / \Gamma_{Y}$ on STEREO A and STEREO B respectively.

[61] Acknowledgments. We thank the team who designed and built the instrument and are very grateful to P.L. Astier for many discussions that helped us to understand the response of the instrument and to evaluate the spacecraft capacitance. The French part was supported by CNES and CNRS. A.C. was supported by Polish Ministry of Science grant NN
203513 038. Several of the authors were supported by NASA grant NNA09DB30A.

[62] Philippa Browning thanks Orville St. Cyr and another reviewer for their assistance in evaluating this paper.

\section{References}

Altobelli, N., S. Kempf, M. Landgraf, R. Srama, V. Dikarev, H. Krüger, G. Moragas-Klostermeyer, and E. Grün (2003), Cassini between Venus and Earth: Detection of interstellar dust, J. Geophys. Res., 108(A10), 8032, doi:10.1029/2003JA009874.

Aubier, M. G., N. Meyer-Vernet, and B. M. Pedersen (1983), Shot noise from grain and particle impacts in Saturn's ring plane, Geophys. Res. Lett., 10, 5-8.

Bale, S. D., P. J. Kellogg, D. E. Larsen, R. P. Lin, K. Goetz, and R. P. Lepping (1998), Bipolar electrostatic structures in the shock transition region: Evidence of electron phase space holes, Geophys. Res. Lett., 25, 2929-2932.

Bale, S. D., et al. (2008), The electric antennas for the STEREO/WAVES experiment, Space Sci. Rev., 136, 529-547.

Bougeret, J.-L., et al. (2008), S/WAVES: The radio and plasma wave investigation on the STEREO mission, Space Sci. Rev., 136, 487-528.

Cully, C. M., R. E. Ergun, and A. I. Eriksson (2007), Electrostatic structure around spacecraft in tenuous plasmas, J. Geophys. Res., 112, A09211, doi:10.1029/2007JA012269.

Czechowski, A., and I. Mann (2010), Formation and acceleration of nano dust in the inner heliosphere, Astrophys. J., 714, 89-99.

Dohnanyi, J. S. (1969), Collisional model of asteroids and their debris, J. Geophys. Res., 74, 2531-2554.

Grün, E., H. A. Zook, H. Fechtig, and R. H. Giese (1985), Collisional balance of the meteoritic complex, Icarus, 62, 244-272.

Grün, E., H. Fechtig, J. Kissel, D. Linkert, D. Maas, J. A. M. McDonnell, G. E. Morfill, G. Schwehm, H. A. Zook, and R. H. Giese (1992), The Ulysses dust experiment, Astron. Astrophys. Suppl. Ser., 92, 411-423.

Gurnett, D. A., E. Grün, D. Gallagher, W. S. Kurth, and F. L. Scarf (1983), Micron-sized particles detected near Saturn by the Voyager plasma wave instrument, Icarus, 53, 236-254.

Gurnett, D. A., W. S. Kurth, L. J. Granroth, and S. C. A. R. L. Poynter (1991), Micron-sized particles detected near neptune by the Voyager 2 plasma wave instrument, J. Geophys. Res., 96, 19,177-19,186.

Gurnett, D. A., J. A. Ansher, W. S. Kurth, and L. J. Granroth (1997), Micron-sized dust particles detected in the outer solar system by the voyager 1 and 2 plasma wave instruments, Geophys. Res. Lett., 24, $3125-3128$.

Henri, P., N. Meyer-Vernet, C. Briand, and S. Donato (2011), Observations of langmuir ponderomotive effects using the Solar Terrestrial Relations Observatory spacecraft as a density probe, Phys. Plasmas, 18, 082308, doi.org/10.1063/1.3622667.

Hsu, H.-W., H. Krüger, and F. Postberg (2012), Dynamics, composition, and origin of Jovian and Saturnian dust stream particles, in Nanodust in the Solar System: Discoveries and Interpretations, Astrophys. Space Sci. Libr., vol. 385, pp. 77-118, Springer, New York, in press.

Kaiser, M. L., T. A. Kucera, J. M. Davila, O. C. S. Cyr, M. Guhathakurta, and E. Christian (2008), The STEREO mission: An introduction, Space Sci. Rev., 136, 5-16.

Kellogg, P. J., S. J. Monson, K. Goetz, R. L. Howard, J.-L. Bougeret, and M. L. Kaiser (1996), Early wind observations of bow shock and foreshock waves, Geophys. Res. Lett., 23, 1243-1246.

Krüger, H., M. Landgraf, N. Altobelli, and E. Grün (2007), Interstellar dust in the solar system, Space Sci. Rev., 130, 401-408.

Kurth, W., T. F. Averkamp, D. A. Gurnett, and Z. Wang (2006), Cassini rpws observations of dust in saturn's e ring, Planet. Space Sci., 54, 988-998.

Malaspina, D. M., I. H. Cairns, and R. E. Ergun (2011), Dependence of Langmuir wave polarization on electron beam speed in type III solar radio bursts, Geophys. Res. Lett., 38, L13101, doi:10.1029/2011GL047642.

Mann, I. (2010), Interstellar dust in the solar system, Annu. Rev. Astron. Astrophys., 48, 173-203.

Mann, I., A. Czechowski, N. Meyer-Vernet, A. Zaslavsky, and H. Lamy (2010), Dust in the interplanetary medium, AIP Conf. Proc., 1216, 491-496.

McBride, N., and J. A. M. McDonnell (1999), Meteoroid impacts on spacecraft: Sporadics, streams, and the 1999 Leonids, Planet. Space Sci., 47, $1005-1013$

Meyer-Vernet, N. (2001), Detecting dust with electric sensors in planetary rings, comets and interplanetary space, paper presented at the 7th International Conference, Eur. Space Agency, Noordwijk, Netherlands.

Meyer-Vernet, N. (2007), Basics of the Solar Wind, Cambridge Univ. Press, New York. 
Meyer-Vernet, N., and C. Perche (1989), Toolkit for antennae and thermal noise near the plasma frequency, J. Geophys. Res., 94, 2405-2415.

Meyer-Vernet, N., M. G. Aubier, and B. M. Pedersen (1986), Voyager 2 at Uranus: Grain impacts in the ring plane, Geophys. Res. Lett., 13, 617-620.

Meyer-Vernet, N., A. Lecacheux, and B. M. Pedersen (1996), Constraints on Saturn's E ring from the Voyager 1 radio astronomy instrument, Icarus, 123, 113-128

Meyer-Vernet, N., A. Lecacheux, and B. M. Pedersen (1998), Constraints on Saturn's G ring from the Voyager 2 radio astronomy instrument, Icarus, 132, 311-320.

Meyer-Vernet, N., M. Maksimovic, A. Czechowski, I. Mann, I. Zouganelis, K. Goetz, M. L. Kaiser, O. C. St.Cyr, J.-L. Bougeret, and S. D. Bale (2009a), Dust detection by the wave instrument on stereo: Nanoparticles picked up by the solar wind?, Solar Phys., 256, 463-474.

Meyer-Vernet, N., A. Czechowski, I. Mann, M. Maksimovic, A. Lecacheux, K. Goetz, M. L. Kaiser, O. C. St.Cyr, S. D. Bale, and G. L. Chat (2009b) Detection of fast nanoparticles in the solar wind, AIP Conf. Proc., 1216, 502-505.

Meyer-Vernet, N., A. Lecacheux, M. L. Kaiser, and D. A. Gurnett (2009c), Detecting nanoparticles at radio frequencies: Jovian dust stream impacts on Cassini/RPWS, Geophys. Res. Lett., 36, L03103, doi:10.1029/ 2008GL036752.
Oberc, P. (1990), Correlations between the Vega 2 plasma wave (APV-N) and dust (SP-1) observations at Comet Halley, Icarus, 86, 314-326.

Oberc, P. (1994), Dust impacts detected by Voyager-2 at Saturn and Uranus: A post-Halley view, Icarus, 111, 211-226.

Oberc, P. (1996), Electric antenna as a dust detector, Adv. Space Res., 17, $105-110$.

Srama, R., et al. (2004), The Cassini cosmic dust analyzer, Space Sci. Rev., $114,465-518$

St.Cyr, O. C., et al. (2009), STEREO secchi and s/waves observations of spacecraft debris caused by micron-size interplanetary dust impacts, Solar Phys., 256, 475-488.

Tsintikidis, D., W. S. Kurth, D. A. Gurnett, and D. D. Barbosa (1995), Study of dust in the vicinity of Dione using the Voyager 1 plasma wave instrument, J. Geophys. Res., 100, 1811-1822.

Tsurutani, B. T., et al. (2003), Dust impacts at Comet P/Borrelly, Geophys. Res. Lett., 30(22), 2134, doi:10.1029/2003GL017580.

Wilck, M., and I. Mann (1996), Radiation pressure forces on "typical" interplanetary dust grains, Planet. Space Sci., 44, 493-499.

Zaslavsky, A., N. Meyer-Vernet, S. Hoang, M. Maksimovic, and S. D. Bale (2011), On the antenna calibration of space radio instruments using the galactic background: General formulas and application to STEREO/ WAVES, Radio Sci., 46, RS2008, doi:10.1029/2010RS004464. 